\title{
Economía del riesgo, un análisis crítico a la mirada de Ulrich Beck
}

\section{Risk economy, a critical analysis of Ulrich Beck's gaze}

\author{
BECK, UlRICH (2006), LA SOCIEDAD DEL RIESGO: HACIA UNA NUEVA MODER- \\ NIDAD, PAIDÓs, BARCELONA, 393 PP., ISBN 84-493-I 892-O.
}

Uno de los trabajos pioneros en temas vinculados al riesgo es la obra del Ulrich, titulada La sociedad del riesgo, hacia una nueva modernidad. Originalmente el autor sugiere una ruptura en el proceso histórico de la modernización a partir del accidente en la central atómica de Chernobyl, en Ucrania. Luego de ese evento, la percepción acerca de los riesgos sufrió una notable transformación. Gran parte de la experiencia de Beck en trabajo de campo se encuentra vinculada al estudio de la familia (Beck, 1985). El autor comprende que las vinculaciones (lazos) familiares están cambiando como producto de la posmodernidad. La desvinculación personal se encuentra asociada a un proceso de individuación -que es irreversible- cuyas consecuencias se ven en la educación y en el trabajo (Beck, 1983).

El accidente nuclear de Chernobyl no sólo conmocionó al mundo, sino que permitió a Beck interrelacionar sus trabajos anteriores con respecto a la disgregación social (una tradición iniciada tiempo atrás por la sociología clásica) con aspectos referidos a la seguridad. En este contexto, las preocupaciones de Beck se emparentan con las de otros pensadores posteriores, como Z. Bauman o R. Castel, en cuyas obras encontramos citas referentes a las contribuciones de Beck. Leer a este autor es adentrarse en las raíces aristotélicas del temor. Para el padre de la Escuela Peripatética, el temor es uno de los actos humanos que tanto por exceso (valentía) o por defecto (cobardía) lleva al ser humano a la reclusión. El temor no comprende ningún fondo y quien todo teme no puede encontrar su compostura. Para Aristóteles, entonces, el temor o miedo es una forma de optimizar el placer evitando el dolor (Aristóteles, Ética, II: 19). En el desarrollo de la presente reseña veremos que mercado y placer se configuran como dos variables obligadas en Beck y sus continuadores.

En los capítulos iniciales de La sociedad del riesgo, Beck se da cuenta que a diferencia de los viajeros medievales, quienes evaluaban los riesgos individuales antes de cada viaje (expresado en forma de aventura), esta nueva percepción del riesgo se presenta como colectiva y catastrófica, ajena a las posibilidades del sujeto. En ese contexto, el autor sostiene que 
dicho cambio se lleva a cabo en concordancia con el aumento de las fuerzas productivas y la materialidad. De esta forma, los riesgos los genera el desarrollo productivo, creando un aumento en las situaciones de peligro en donde la antigua estratificación social no es suficiente. En efecto, el autor sugiere que el quiebre ha dado origen a una sociedad del riesgo, la cual en oposición con la sociedad de la riqueza reparte riesgos entre ricos y pobres.

De esta forma, el análisis de Beck lleva a suponer que la percepción del riesgo se encuentra vinculada a una necesidad de consumo. Por ende, no rompe de ninguna manera el desarrollo capitalista sino que lo expande. Los bienes de consumo, ingresos y riquezas se reparten en tanto que son recursos escasos que generan una brecha entre los diferentes grupos sociales. A la lógica de la apropiación se le impone su contrario, la negación. Por ese motivo, los daños colaterales del consumo excesivo y no sustentable son negados, puestos en duda o censurados por los grupos privilegiados que sustentan dichas prácticas y finalmente por toda la población. No obstante, en tanto efectos reales, la globalización y generalización de las responsabilidades exime a quienes tienen control directo de los medios y modos de producción. En otros términos, se concreta una unidad entre víctima y victimario. $\mathrm{Al}$ respecto, Beck explica que en la clásica sociedad de clases, los grupos basaban sus distinciones en el estilo social al cual estaban atados desde su ingreso a la sociedad, los riesgos eran, entonces, conocidos y experimentados por los propios medios; por el contrario, en la sociedad del riesgo, éstos se encuentran dados por medios externos al sujeto, como pueden ser el campo científico o el periodismo. En parte, el ciudadano comienza a perder una importante porción de su soberanía cognitiva; así, surgen los conocidos expertos del riesgo, quienes junto a las estructuras económicas ejercen una explotación comercial con miras a nuevos mecanismos de seguridad.

Básicamente, la preocupación de Beck está ligada a la ética del medio ambiente y a la degradación constante de los desechos tóxicos producto del capitalismo industrial. Según su posición inicial, esta nueva forma de concebir la modernidad obliga a las sociedades a unirse en vistas de un riesgo que la mayoría de las veces excede las posibilidades individuales y se presenta como externo. En su propia dinámica de desarrollo democratizan los peligros desdibujando las fronteras del Estado-nación, pero a la vez pone nuevas fuerzas en conflicto y consenso. En el transcurso del pasaje de un tipo de sociedad a otro, dice Beck,

Comienza a cambiar la cualidad de la comunidad. Dicho esquemáticamente, en estos dos tipos de sociedades modernas se abren paso sistemas axiológicos completamente distintos. Las sociedades de clases quedan referidas en su dinámica de desarrollo al ideal de la igualdad [...] no sucede lo mismo con la sociedad del riesgo. Su contraproyecto normativo, que está en su base y la estimula, es la 
seguridad. En lugar del sistema axiológico de la sociedad desigual aparece, pues, el sistema axiológico de la sociedad insegura (p. 69).

Este clima de inseguridad creciente transforma la forma de concebir la reciprocidad en la comunidad; la jerarquización por clase llevaba implícita la idea de alcanzar una meta, mientras que la sociedad del riesgo tiende a evitar lo peor; en otros términos, la idea de participación se reemplaza por la de protección, dando origen a la comunidad del miedo.

En capítulos sucesivos sugiere la presencia de una modernidad reflexiva, Beck enfatiza que la producción de los riesgos es inversamente proporcional a la distribución de la riqueza. Su tesis principal apunta a que mientras en la sociedad industrial la dinámica en la producción material domina a la de riesgos, en las sociedades actuales esa relación se invierte. La ganancia técnico-económica se encuentra eclipsada. Por supuesto, estos peligros no se circunscriben a acciones individuales, lugares o grupos específicos, sino a la humanidad toda. Sin embargo, los sistemas parecen sacar provecho de las propias necesidades, y no es extraño que el riesgo implique un impulso económico de primer rango para toda la estructura social. Las irregularidades producidas por el propio capitalismo se reinventan funcionalmente en provecho del consumo masivo.

Las necesidades humanas poseen una limitación, una vez satisfechas se requiere de un tiempo para su nueva reactivación. En cambio, según Beck, la imposición ideológica del riesgo implica un pozo de necesidades sin fondo que nunca se satisface. Aclarado esto, los miedos no sólo irán en ascenso sino que se prolongan según los intereses del mercado. "Así pues, con la imposición de la sociedad del riesgo, producción y consumo serán elevados a una escala completamente nueva. En lugar de las necesidades dadas de antemano y manipulables, como punto de referencia de la producción de mercancías, se sitúa el riesgo autoproducible" (p. 79).

Esta dinámica autorreferencial es independiente del peligro real y de sus causas, mas presenta productos acabados para hacer frente a los riesgos, pero no reducen totalmente la ansiedad, y en consecuencia no proponen políticas reales de prevención. La idea central en Beck con respecto al tema, es que la sociedad moderna tiende a producir en forma sistemática la propia amenaza mediante la explotación productiva de sus propios riesgos; una situación comparable históricamente con el pasaje del feudalismo al capitalismo en la Edad Media. En otras palabras, la propia producción genera limitaciones y riesgos, los cuales lejos de desaparecer dan lugar a su manipulación simbólica y por medio de ella a la producción de medios orientados a la protección y a la perpetuación. Pero paradójicamente, como resultado final, se genera un quiebre en las formas productivas que dan nacimiento a una nueva estructura. 
Hasta aquí hemos intentado presentar lo más fielmente posible el pensamiento de Beck con respecto a la obra de referencia. Por lo demás, su problema principal radica en que no profundiza en cuáles son los factores que han determinado el quiebre de la sociedad productiva de clase a la de riesgo. Aun cuando su descripción es muy clara en cuanto a las consecuencias y efectos del fenómeno, se observa una contradicción con respecto a la construcción teórica de una modernidad reflexiva. A saber, si la propia sociedad, en sus palabras, se nutre de los propios riesgos que genera con el fin de reproducirse, la cuestión central es ipor qué implícitamente desaparece? Esta situación paradójica oscurece el panorama interpretativo de la propia teoría de Beck. Mas por lo pronto, la obra en cuestión se configura como uno de los trabajos pioneros y más interesantes en cuestiones relacionadas con el riesgo, el temor y los estadios de psicosis colectivas de los cuales son presa las sociedades modernas.

A lo largo de su carrera académica, Ulrich Beck ha cosechado seguidores y detractores en cuanto a su teoría del riesgo y la sociedad. Para R. Castel, por ejemplo, las sociedades modernas equipadas con todo tipo de bienes materiales y protecciones, son aquéllas donde el sentimiento de inseguridad no sólo es moneda corriente, sino que atraviesa todos los estratos sociales. En concordancia con los postulados aristotélicos, esta compleja situación lleva a R. Castel a plantear una hipótesis por demás interesante: la inseguridad moderna no sería la ausencia de protecciones o medios, sino todo lo contrario, una obsesiva manía vinculada a la búsqueda incesante de seguridad en un mundo social interrelacionado. La tesis principal de Castel es que desde 1980 se ha instalado en la sociedad un nuevo problema con respecto a la inseguridad; un aumento en los canales de incertidumbre y una especie de malestar frente al porvenir quedan subordinados a que ocurran posibilidades inverosímiles de manera compensatoria (Castel, 2006: 78). Castel sigue el argumento de Beck cuando dice que la modernidad ha generado un vaciamiento de las estructuras económico-políticas, relegando al Estado a una posición subordinada con respecto al mercado y las demandas del ciudadano.

En Z. Bauman también existe un expreso reconocimiento a los aportes de Beck cuando reafirma la existencia de una modernidad (líquida) responsable de la escalada constante de temor en las sociedades industriales. Para Bauman, el riesgo es un mecanismo por el cual el individuo intelectualiza lo imprevisible que acecha en lo trágico, reduce la angustia de lo incognoscible. En la sociedad líquida de consumidores la estrategia consiste en marginar y menospreciar todas aquellas cosas que tienen una duración longeva. Los miedos han acompañado a los hombres en toda su historia, pero es en la modernidad líquida cuando son netamente intercambiables por mercancías. Estas fuerzas sociales se ubican por encima de los Estados 
dejándolos impotentes en la protección de sus propios ciudadanos; por otro lado es la misma competencia del mercado el factor que desencadena la posterior desconfianza y falta de solidaridad entre los hombres. Esta no conexión deriva en un sentimiento paranoico de supuesta agresión (Bauman, 2008).

Por el contrario, para Luhmann los sistemas que alcanzan cierta complejidad pueden ver el futuro como espejo del pasado y orientarse semióticamente. El riesgo es el producto de la contingencia de la decisión del sujeto con arreglo a una utilidad estimada. Su tesis enfatiza que la sociedad moderna representa el futuro como riesgo para superar la paradoja del tiempo. En este contexto, Beck y sus seguidores confunden el riesgo propio de los sistemas decisorios con la amenaza. La caída de un avión o un terremoto no son un riesgo para el ciudadano en general porque su decisión no determina el evento en ninguna de sus formas; las amenazas no se forman por riesgos individuales. Según Niklas Luhmann, Beck además equivoca su desarrollo del riesgo por dos motivos centrales. En primer lugar, éste se encuentra más preocupado por alarmar a la sociedad con cómo riesgos no percibidos se pueden convertir en reales amenazas, que en comprender el fenómeno. Segundo, el riesgo no sólo está vinculado con la racionalidad técnica, sino con la posibilidad de ejercer la decisión en una realidad contingente. El riesgo se configura como tal siempre y cuando pueda ser evitable por el sujeto. Luhmann propone que el riesgo no puede ser reducido a menos que se disminuya la posibilidad del sujeto de decidir, lo cual es incorrecto (Luhmann, 2006: 74).

Otro de los puntos polémicos en el argumento de Beck es su énfasis en la lógica cognitiva. A la idea apocalíptica de vaciamiento de las estructuras, Lash y Urry proponen que la posmodernidad trae consigo una reflexivilidad que profundiza y abre muchas alternativas positivas para los vínculos sociales, como la intimidad, la amistad o el ocio. Uno de los problemas que tiene Beck en su análisis de la reflexivilidad es trivializar el papel de la estética y centrar su trabajo exclusivamente en lo cognitivo. La lectura de Beck enfatiza que existen tres estadios que vinculan la producción material a los riesgos: preindustrial, industrial y del riesgo. Si se parte de la base que las sociedades preindustriales no producían riesgos sino que vivían también las amenazas de la naturaleza, éstas no eran directamente provocadas por el progreso técnico. Tomando las influencias nietzscheanas, Lash y Urry sugieren que la resubjetivización propia de este proceso ha llevado a una dicotomización de las estructuras vigentes que obligan a redefinir el papel del sujeto como entidad inserta en el mundo. En un mundo que desafía la lógica del mundo apolíneo, un mundo que es amoral y natural por sí mismo. Las transacciones comerciales, las jerarquías empresariales, las modas se han transformado y se hacen más horizontales que en épocas 
anteriores. La alegoría ha reemplazado al símbolo en un horizonte acronológico que desdibuja la tradición y la historia. A la artificialidad propia del símbolo se le contrapone la profundidad de la alegoría y el sentido. Es decir, lejos de tratarse de un vaciamiento total como sugiere Beck, Lash y Urry consideran la posmodernidad como un proceso que construye sus cimientos en la experiencia individual del ser-en-el-mundo (Lash y Urry, 1998). La posmodernidad ha erosionado las bases jerárquicas lineales propias de la modernidad generando una hermenéutica del sujeto. Los espacios comienzan a desdibujarse subsumidos en lo abstracto del signo.

Una vez expuestos los argumentos precedentes de Beck en perspectiva con Bauman, Castel, Luhmann, Lash y Urry, la hipótesis de trabajo que puede despredenderse del análisis del presente texto sugiere que la percepción de riesgo es mayor en aquéllos económicamente activos en comparación con otros grupos laboralmente pasivos, como menores de edad y jubilados o pensionados. En este sentido, el riesgo se configura como un fenómeno propio de los grupos insertos en el sistema de consumo. Quizás a su manera, Beck haya expresado -sin saberlo- el fuerte vínculo que el milenarismo tiene sobre las sociedades y sus estructuras económicas. Empero, ese es motivo de un segundo trabajo de revisión.

\section{Bibliografía}

Aristóteles (1997), Ética Nicomaquea, Porrúa, México.

Bauman, Zygmunt (2008), Miedo liquido: la sociedad contemporánea y sus miedos líquidos, Paidós, Buenos Aires.

Beck, Ulrich (1983), “Jenseits von Stand und Klasse?”, en R. Kreckel (comp.), Soziale Ungleichheiten, vol. esp. 2, Sozialen Welt, Gotinga, pp. 602-617.

Beck, Ulrich (1985), "Von der Verganglichkeit der Industriegesellschaft", en Th. Schmid (comp.), Das Pfeifende Schwein, Berlín.

Castel, Robert (2006), La inseguridad social: ¿qué es estar protegido?, El Manantial, Buenos Aires.

Lash, Scott y John Urry (1998), Economias de signo y espacio: sobre el capitalismo de la posorganización, Amorrortu, Buenos Aires.

Luhmann, Niklas (2006), Sociología del riesgo, Universidad Iberoamericana, México. 
Recibida: 17 de abril de 2009. Aceptada: 27 de octubre de 2009.

Maximiliano Korstanje. Es licenciado en turismo por la Universidad de Morón, Argentina, y candidato a doctor en psicología social por la Universidad John F. Kennedy, Argentina. Es autor de más de 60 artículos, ensayos y trabajos científicos vinculados a la historia, la filosofía, la antropología, la psicología, la economía y la sociología del turismo. En la actualidad se desempeña como docente en la Universidad de Palermo (Argentina), es miembro del Comité de Redacción de Turydes: Revista de Investigación en Turismo y Desarrollo Local, Grupo Eumed.net, Universidad de Málaga, España. Es investigador miembro de la Asociación Internacional de Expertos Científicos en Turismo (Suiza) y se desempeña como evaluador ad-honorem de las revistas académicas Rbtur (revista brasileña de pesquisa en turismo), Santiago (Universidad de Oriente, Cuba), Estudios de Ciencias Sociales (Universidad de Guadalajara, México), Gestión turística (Universidad Austral de Chile) y Cuadernos en Turismo (Universidad de Murcia, España). 